\title{
Exercise in Type 1 (insulin-dependent) diabetic patients treated with continuous subcutaneous insulin infusion
}

\section{Prevention of exercise induced hypoglycaemia}

\author{
G. E. Sonnenberg, F. W. Kemmer and M.Berger \\ Department of Nutrition and Metabolism, (WHO Collaborating Center for Diabetes), Heinrich-Heine Universität, Düsseldorf, FRG
}

\begin{abstract}
Summary. The study was performed to investigate the effects of mild to moderate exercise on blood glucose levels, metabolite concentrations and responses of counterregulatory hormones in tightly controlled Type 1 (insulin-dependent) diabetic patients treated by continuous subcutaneous insulin infusion, and to quantify the measures necessary to prevent acute and late exercise-induced hypoglycaemia. Seven male patients started a 60 min exercise period $90 \mathrm{~min}$ after an insulin bolus and a standard breakfast; they were monitored during a post-exercise resting period of $5 \mathrm{~h}$ $30 \mathrm{~min}$. Different basal and premeal insulin infusion rates were applied. (Near)normoglycaemia prevailed throughout the study during the control protocol when the subjects did not exercise and received their usual insulin dose. When they exercised without changing the insulin dose, four patients were forced to stop due to hypoglycaemia. This effect of exercise could be attenuated but not completely avoided if the basal infusion rate of insulin was discontinued during the exercise period. The pronounced increase in catechol-
\end{abstract}

amine and growth hormone concentrations during exercise were not sufficient to prevent hypoglycaemic reactions. Hypoglycaemia during exercise could only be prevented when the premeal insulin bolus was reduced by $50 \%$ in addition to the discontinuation of the basal insulin infusion during exercise. In order to reduce late hypoglycaemic reactions after exercise the best measure proved to be a reduction of the basal insulin infusion rate by $25 \%$ during post-exercise hours. Administration of only $50 \%$ of the basal insulin infusion rate during this time was associated with blood glucose levels being raised up to $8 \mathrm{mmol} / \mathrm{l}$. In conclusion, Type 1 diabetic patients treated with continuous subcutaneous insulin infusion at (near)normoglycaemia need to reduce their insulin dosage before, during, and after mild to moderate endurance exercise in order to minimize the risk of acute and late hypoglycaemia.

Key words: Exercise, Type 1 (insulin-dependent) diabetes, CSII, hypoglycaemia.
Healthy subjects usually do not have to fear any metabolic complications during physical activity. Diabetic patients, however, are faced with the risk of exercise induced hypoglycaemia or under certain conditions severe ketoacidosis [1]. Hypoglycaemia develops during physical activity because the physiological glucoregulation, $i$. e. an increase in hepatic glucose production precisely matching the enhanced fuel demands of the working muscle, is impaired in Type 1 (insulin-dependent) diabetic patients $[2,3]$. The main reason for this defect in glucoregulation during exercise is the hyperinsulinaemia which usually prevails in insulin treated patients $[4,5]$. The most logical measure to prevent exercise induced hypoglycaemia in diabetic patients is to mimic the physiological reduction of insulin secretion occurring in non-diabetic subjects during exercise [6]. In patients with conventional insulin treatment this can be achieved by a drastic reduction of the insulin dose prior to or even after exercise as we [7] and others have shown [8] previously. As a consequence, certain guidelines for prevention of exercise induced hypoglycaemia in patients on conventional insulin treatment have been proposed $[6,9]$.

In principle, patients with continuous subcutaneous insulin infusion (CSII) therapy should also follow guidelines such as reduction of the insulin bolus in anticipation of postprandial exercise [10] and adaptation of the insulin infusion rate [11]. However, most previous studies on exercise and CSII [12-16] were performed to compare the metabolic and hormonal effects of exercise in patients on CSII therapy rather than to investigate measures to prevent exercise induced metabolic complications. In order to estimate the insulin dose reduction necessary to prevent acute and/or late hypoglycaemic reactions in CSII treated Type 1 diabetic patients with (near)normoglycaemia, we have studied the effects of various basal insulin infusion rates both with and without a reduced premeal in- 


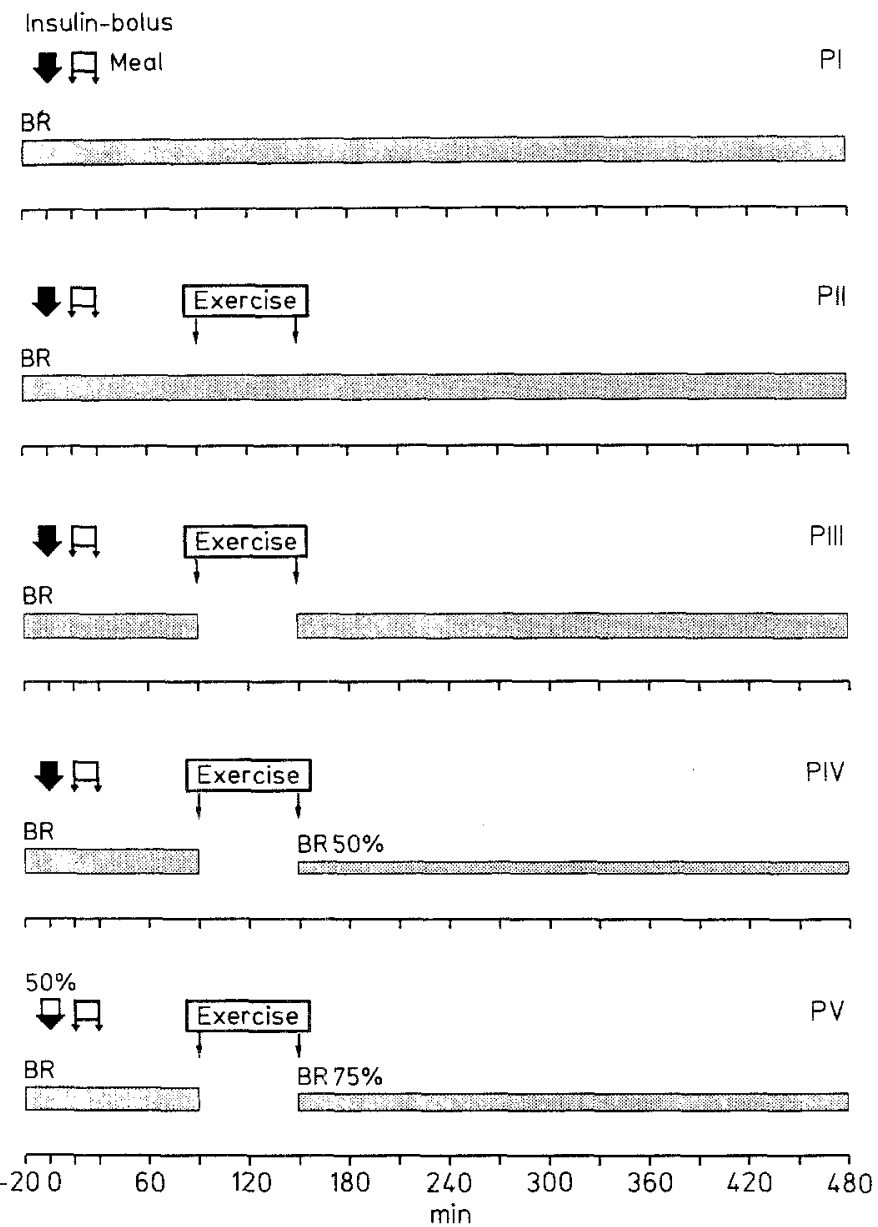

Fig. 1. Diagram of five different experimental protocols $\left(\mathrm{P}_{\mathrm{r}}-\mathrm{P}_{\mathrm{v}}\right)$ showing mealtime and modifications of exercise, basal insulin infusion rate (BR) and insulin bolus. (For further details see text)

sulin bolus on glucose homeostasis in a group of CSII treated diabetic patients performing exercise of a defined duration and intensity.

\section{Subjects and methods}

\section{Subjects}

Seven male, physically untrained, C-peptide negative Type 1 diabetic patients treated with CSII were recruited from our outpatient clinic and volunteered to participate in the study after giving their written informed consent. The study was approved by the Ethical Committee of the Medical Faculty of the University of Dusseldorf according to the Declaration of Helsinki. Further clinical and laboratory data are given in Table 1. Except for two patients with background retinopathy all patients were free of diabetic late complications as assessed by ophthalmoscopy, creatinine clearance, proteinuria and clinical examination. The patients were otherwise healthy and received no medication other than insulin. The patients had been on CSII treatment for 4 to 17 months and maintained normoglycaemia or near normoglycaemia as indicated by home blood glucose monitoring and $\mathrm{HbA}_{1 c}$ levels (normal range 4.1-7.8\%). The individual insulin doses are given in Table 1. Purified porcine regular insulin (Actrapid, Novo Industri, Copenhagen, Denmark) was infused using an Auto Syringe pump AS $6 \mathrm{C}$ (Auto Syringe Inc., Hookset, New Hampshire, USA) via a 25 gauge metal tip catheter
(Venofix, Braun Melsungen, FRG) inserted into the subcutaneous tissue of the anterior abdominal wall in a way that excluded intramuscular infusion.

\section{Experimental design}

Each patient participated in five different protocols $\left(\mathrm{P}_{\mathrm{I}}-\mathrm{P}_{\mathrm{V}}\right)$ with an interval of at least 10 days between each experiment. Insulin infusion rates and exercise were varied in the five protocols as shown in Figure 1, while all other conditions were kept constant. All subjects started with $P_{1}$ which served as the control experiment with the usual insulin dose but without exercise. $\mathrm{P}_{\mathrm{II}}-\mathrm{P}_{\mathrm{IV}}$ were performed in random order, in $\mathrm{P}_{\mathrm{II}}$ the insulin rates were not changed and leg exercise was performed for $60 \mathrm{~min}$ on a cycle ergometer. In $P_{I I I}$ and $P_{I V}$ the basal insulin infusion rate was discontinued during exercise and thereafter, insulin was infused at $100 \%\left(\mathrm{P}_{\mathrm{III}}\right)$ and $50 \%\left(\mathrm{P}_{\mathrm{IV}}\right)$ of the initial basal rate until the end of the experiment. The results of the blood glucose concentrations obtained in these protocols led to the design of $\mathrm{P}_{\mathrm{V}}$. In this protocol the premeal insulin bolus was reduced by $50 \%$, the basal rate was discontinued during exercise and insulin was infused at $75 \%$ of the initial rate during the post exercise period.

\section{Experimental procedures}

The patients reported to the laboratory at 07.30 hours after an overnight fast. Studies were initiated only if fasting blood glucose was in the normal range and no hypoglycaemic reactions had occurred during the preceding night. For this purpose the patients had to monitor blood glucose values at bed-time of the preceding day, at 03.00 hours, and on the morning of the experiment. On arrival in the laboratory a 17 gauge teflon cannula was inserted into an antecubital vein for blood sampling, and was kept patent by infusion of $\mathrm{NaCl}$ $(154 \mathrm{~mol} / \mathrm{l})$. After $30 \mathrm{~min}$ of resting in a supine position and with drawal of basal blood samples the patients administered the usual premeal insulin bolus $\left(50 \%\right.$ in $\left.\mathrm{P}_{\mathrm{V}}\right)$. The insulin dose varied among patients but was identical for all protocols. The patients ate a standard breakfast 15 min later containing approximately $48 \mathrm{~g}$ carbohydrate, $20 \mathrm{~g}$ fat and $20 \mathrm{~g}$ protein. The meal was consumed over a $15 \mathrm{~min}$ period. Exercise was started $90 \mathrm{~min}$ after administration of the insulin bolus and continued for $60 \mathrm{~min}$. The patients exercised on a vertical cycle ergometer (Conditronic 30, Keiper Dynavit, Rockenhausen, FRG) allowing continous monitoring of pulse rate by a pulsemeter fixed to one ear lobe. On the basis of continously adjusting to the preset pulse rate of 110 to 120 per min the patients performed a workload of an average of 80 watt. The workload did not differ between the five protocols and corresponded to a calculated $0_{2}$ intake of $1.31 / \mathrm{min}$ which can be classified as mild to moderate exercise intensity [17]. The subsequent post-exercise period was $5 \mathrm{~h}$ $30 \mathrm{~min}$; thus the total experimental time span of each protocol was $8 \mathrm{~h}$ and $20 \mathrm{~min}$. Before and after exercise the patients rested in a supine position. Except for breakfast all subjects abstained from

Table 1. Median and range of clinical data of seven Type 1 (insulin dependent) diabetic patients

\begin{tabular}{lll}
\hline Age (years) & 20 & $(15-31)$ \\
Duration of diabetes (years) & 9 & $(7-14)$ \\
Body mass index $\left(\mathrm{kg} / \mathrm{m}^{2}\right)$ & 22.1 & $(20.6-24.0)$ \\
Blood pressure $(\mathrm{mm} \mathrm{Hg})$ & $120 / 80$ & $(110 / 60-140 / 90)$ \\
Creatinine clearance $(\mathrm{ml} / \mathrm{min})$ & 110 & $(90-125)$ \\
Proteinuria $(\mathrm{mg} / 24 \mathrm{~h})$ & 40 & $(18-101)$ \\
HDL-cholesterol $(\mathrm{mmol} / \mathrm{l})$ & 1.43 & $(1.17-1.72)$ \\
Triglyceride $(\mathrm{mmol} / \mathrm{l})$ & 0.70 & $(0.53-0.97)$ \\
Insulin basal rate $\left(\mathrm{U} \cdot \mathrm{kg}^{-1} \cdot 24 \mathrm{~h}\right)$ & 0.32 & $(0.24-0.57)$ \\
Insulin bolus $(\mathrm{U} / 10 \mathrm{~g} \mathrm{CHO})$ & 1.4 & $(1.0-1.5)$ \\
$\mathrm{HbA}_{\mathrm{lc}}(\%)$ & 7.3 & $(6.6-8.0)$ \\
\hline
\end{tabular}



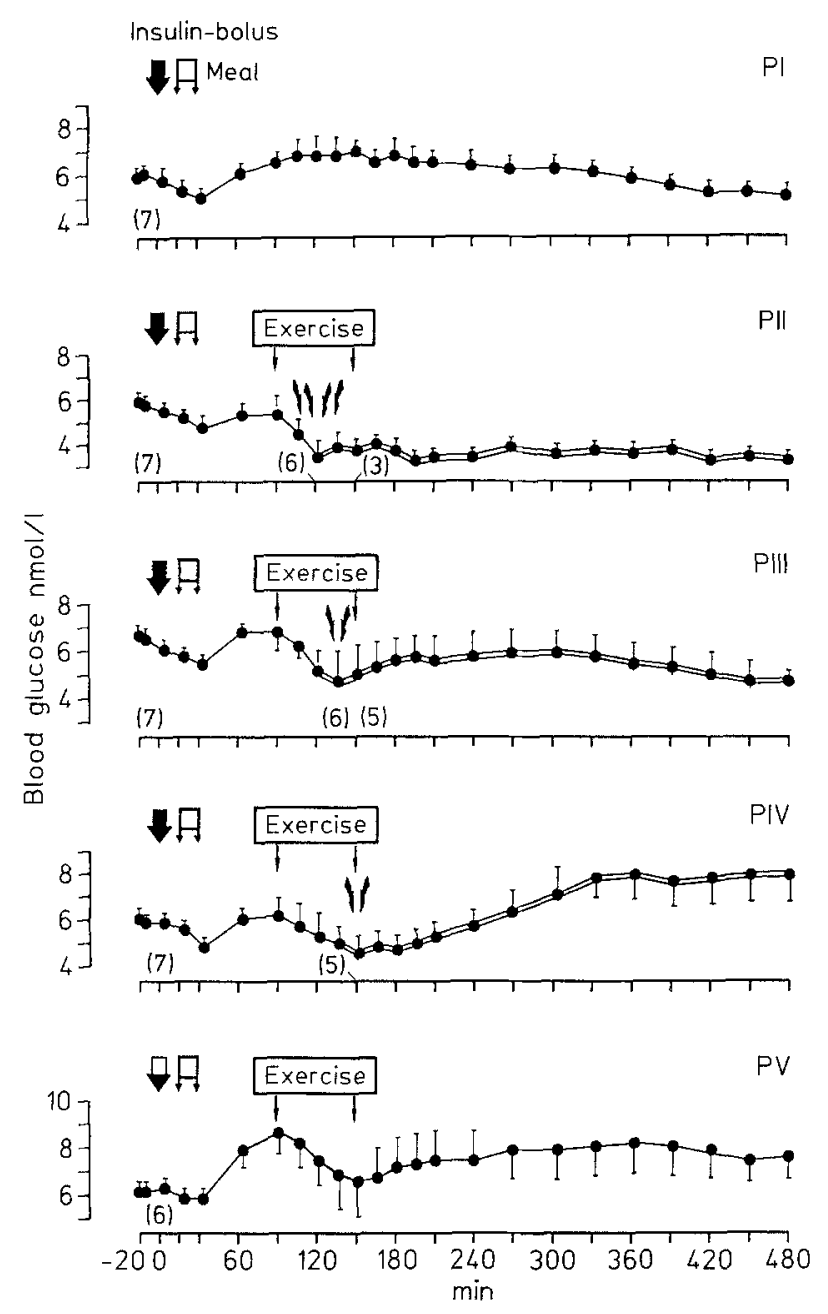

Fig. 2. Blood glucose concentrations of continuous subcutaneous insulin infusion (CSII) treated Type 1 (insulin-dependent) diabetic patients before, during and after mild to moderate exercise in the five different protocols. Curved arrows indicate time and number of patients dropping out due to hypoglycaemia. Figures in brackets indicate number of patients continuing the protocol. Values are given as mean $\pm \mathrm{SEM}$ indicated by vertical bars

food, caffeinated beverages and smoking throughout the experiment. An experiment was discontinued when blood glucose fell to $2.5 \mathrm{mmol} / 1$ or less. The discontinuation of the basal insulin infusion during exercise was achieved by removing the battery from the insulin pump.

\section{Analytical procedures}

Thirty minutes after the insertion of the intravenous cannula, blood samples were taken for base-line measurements at $-20,-15$ and $0 \mathrm{~min}$. Thereafter, blood samples were taken every $15 \mathrm{~min}$ for instant blood glucose measurements, using the Beckman Glucose Analyzer (Beckman Instruments, Fullerton, Calif., USA). At intervals indicated in all Figures and Tables additional blood samples were collected into chilled tubes. One portion of whole blood was immediately added to ice-cold $2 \mathrm{~N} \mathrm{HClO}_{4}$, and the neutralized supernatant was assayed for pyruvate, acetoacetate and $\beta$-hydroxybutyrate as previously described [1]. Serum lactate was determined by an enzymatic-amperometric method [18]. Another portion of blood was transferred into chilled tubes containing EDTA-Na $\mathrm{Na}_{2}$ and aprotinine. After centrifugation plasma was frozen at $-20^{\circ} \mathrm{C}$ for later determi- nation of glucagon by radioimmunoassay with use of $30 \mathrm{~K}$ antiserum (kindly provided by Dr. R. Unger, Dallas, Tex USA). Plasma catecholamine levels were analysed by a radioenzymatic assay [19] from deproteinized plasma stored at $-70^{\circ} \mathrm{C}$. A fourth portion of the blood was centrifuged and the serum stored at $-20^{\circ} \mathrm{C}$ for analysis of serum concentrations of non-esterified fatty acids [20], free insulin [21], growth hormone and cortisol [1].

\section{Statistical analysis}

Results are expressed as means \pm SEM. For comparison within the same protocol, the paired $t$-test was used. Analysis of variance was used to compare the various test periods (base-line, meal, exercise and post-exercise) of the five protocols. Post hoc analysis for testing differences between two protocols was performed using the Duncan $t$-test.

\section{Results}

Blood glucose concentrations and serum free insulin levels

In the control protocol, mean base-line blood glucose concentration (Fig. 2) was $6.1 \pm 0.2 \mathrm{mmol} / \mathrm{l}$. After insulin bolus administration, it fell slightly and was followed by a postprandial increase reaching a maximum of $7.2 \pm 0.7 \mathrm{mmol} / 1$ at $150 \mathrm{~min}$. Thereafter, blood glucose declined continuously to a mean value of $4.3 \pm 0.6 \mathrm{mmol} / \mathrm{l}$ at $480 \mathrm{~min}$. Comparing the five protocols, no significant differences were observed in base-line blood glucose concentrations $(F(4,29)=0.59)$ and in mean values calculated over the postprandial period $(15-90 \mathrm{~min} ; \mathrm{F}(4,29)=2,18)$; however, the 90 min value in protocol $P_{\mathrm{V}}(9.0 \pm 1.1$ mmol/l) was significantly higher than in the other proto$\operatorname{cols}(\mathrm{F}(1,29)=2.31 ; p<0.05)$.

A substantial decrease in the blood glucose concentration was observed in all protocols during the second half of the exercise period. In protocol $P_{\text {II }}$ four patients had to terminate exercise due to blood glucose values of $2.5 \mathrm{mmol} / \mathrm{l}$ or below. In the three patients who completed the protocol the blood glucose concentrations remained between 3.3 and $3.9 \mathrm{mmol} / 1$ until the end of the study. In protocol $\mathrm{P}_{\text {III }}$, only two patients had to interrupt the study during exercise due to low blood glucose concentrations. After exercise, glycaemia of the remaining five patients first rose slightly to a maximal value of $6.2 \pm 1.0 \mathrm{mmol} / \mathrm{l}$ at $270 \mathrm{~min}$ and, thereafter, decreased to $5.0 \pm 0.7 \mathrm{mmol} / \mathrm{l}$ at $480 \mathrm{~min}$. In protocol $\mathrm{P}_{\mathrm{IV}}$, again two patients developed hypoglycaemic values and had to interrupt exercise, whereas the post-exercise period of the remaining five patients who now received only $50 \%$ of their usual basal insulin infusion rate in accordance with the experimental protocol ended with a blood glucose increase to a maximum of $8.4 \pm 1.2 \mathrm{mmol} / 1$. Only in protocol $\mathrm{P}_{\mathrm{V}}$ where the pre-meal insulin bolus - reduced by $50 \%$ - resulted in an elevated pre-exercise blood glucose level, no hypoglycaemia was observed. When a basal rate reduced by $25 \%$ was infused during the post-exercise period, glycaemia increased to $8.6 \pm 1.2 \mathrm{mmol} / \mathrm{l}$ at $360 \mathrm{~min}$ and fell slightly toward $8.0 \pm 0.9 \mathrm{mmol} / 1$ at the end of the protocol. During the post-exercise period, blood glucose concentrations were 

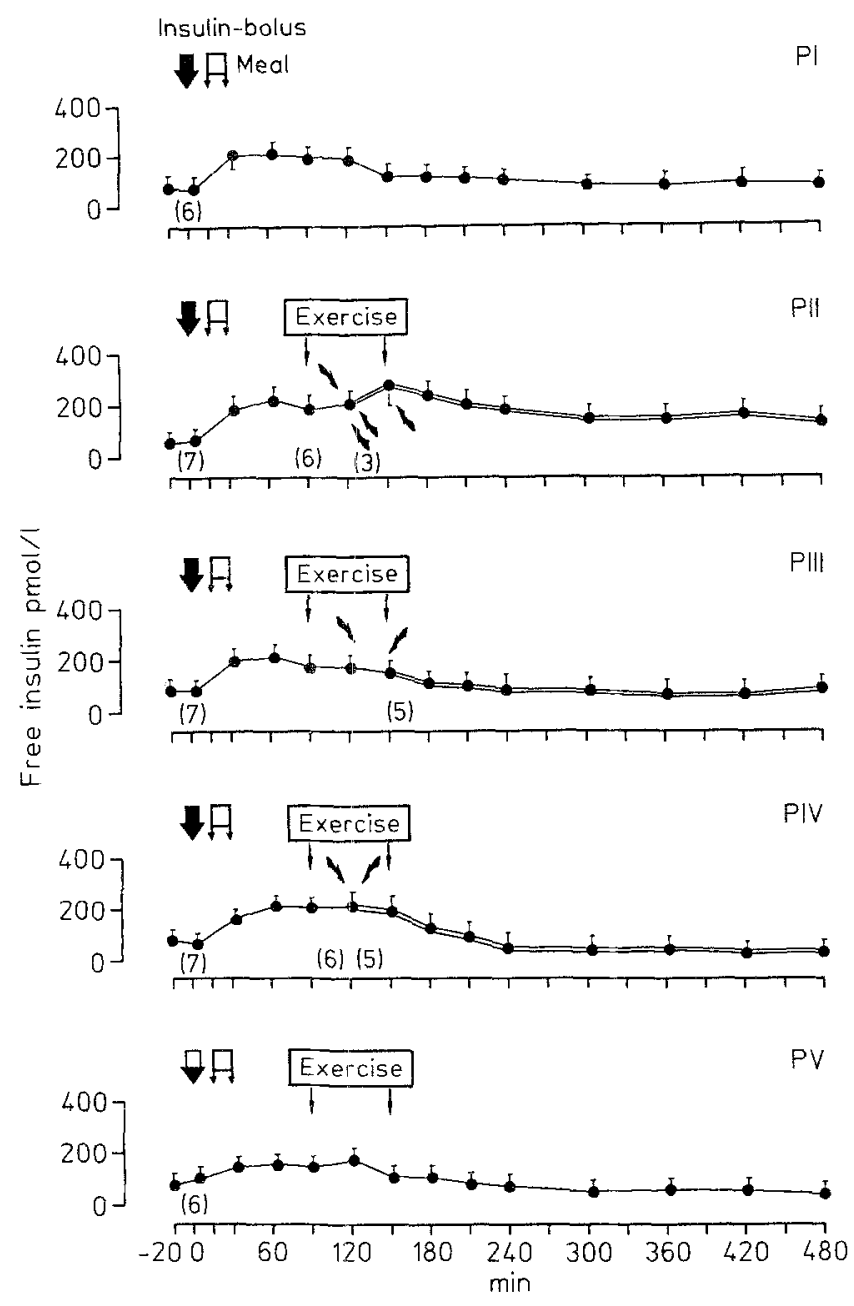

Fig. 3. Serum free insulin concentrations of CSII treated Type 1 diabetic patients before, during and after mild to moderate exercise in the five different protocols. Symbols as in Fig. 2

significantly different between the four protocols $P_{\text {II }}$ to $P_{V}$ when the maximal levels of each protocol were compared $(\mathrm{F}(3,15)=3.55, p<0.05)$. These differences were mainly due to low blood glucose concentrations in $\mathrm{P}_{\mathrm{II}}$ and elevated values in $P_{I V}$ and $P_{V}$.

Under control conditions $\left(\mathrm{P}_{\mathrm{I}}\right)$, the mean base-line concentration of serum free insulin was $89 \pm 14.4 \mathrm{pmol} / \mathrm{l}$ (Fig.3); 30 min after administration of the insulin bolus, an increase of insulin levels to a mean of $225 \pm 25.8 \mathrm{pmol} / \mathrm{l}$ occurred which was followed by a plateau over $60 \mathrm{~min}$ and by a decrease to $146.5 \pm 10.7 \mathrm{pmol} / \mathrm{l}$ at $180 \mathrm{~min}$. Thereafter, mean serum free insulin fell slightly and consistently reaching nearly base-line concentrations at $480 \mathrm{~min}$. Whereas no significant differences between the five protocols were obtained in base-line insulin levels $(F(4,29)=0.65)$, the increase after bolus administration was significantly different $(\mathrm{F}(4,29)=3.77, p<0.05)$ which was caused mainly by low values in $\mathrm{P}_{\mathrm{V}}$ in accordance with the experimental protocol. In $\mathrm{P}_{\mathrm{II}}$ the continuous administration of the basal insulin rate during exercise was associated with a further increase of serum free insulin in three out of six patients during the first $30 \mathrm{~min}$ of exercise. In those three patients who were able to continue exercise without hypoglycaemia, serum insulin concentrations increased even further at a range two- to threefold higher than the base-line values until the end of the study. In protocol $P_{I I I}$ and $P_{y}$, nearly identical values of free insulin were obtained after exercise when compared with the control protocol. The last $3 \mathrm{~h}$ of $\mathrm{P}_{\mathrm{IV}}$ were characterized by low free insulin concentrations ranging between 36 and $59 \mathrm{pmol} / \mathrm{l}$. Comparing the insulin concentrations of all five protocols, the analysis of variance revealed significant differences in maximal and mean concentrations between the protocols over the post-exercise period $(\mathrm{F}(4,29)=4.23$, and $\mathrm{F}(4,29)=5.40, p<0.05)$.

\section{Metabolic variables}

Mean postabsorptive blood concentrations of ketone bodies (acetoacetate and $\beta$-hydroxybutyrate; Table 2) were at 209 and $249 \mu \mathrm{mol} / \mathrm{l}$ respectively in the control protocol and did not differ significantly from the values of $P_{\mathrm{II}}$ to $P_{\mathrm{V}}$. After the insulin bolus and breakfast, a decrease

Table 2. Mean \pm SEM blood concentrations of ketone bodies (acetoacetate plus $\beta$-hydroxybutyrate) expressed in $\mu \mathrm{mol} / 1$

\begin{tabular}{rlllll}
\hline $\begin{array}{l}\text { Time } \\
\text { Min }\end{array}$ & $\begin{array}{l}\mathrm{P}_{\mathrm{I}} \\
n=7\end{array}$ & $\begin{array}{l}\mathrm{P}_{\mathrm{II}} \\
n=7\end{array}$ & $\begin{array}{l}\mathrm{P}_{\mathrm{III}} \\
n=7\end{array}$ & $\begin{array}{l}\mathrm{P}_{\mathrm{IV}} \\
n=7\end{array}$ & $\begin{array}{l}\mathrm{P}_{\mathrm{V}} \\
n=6\end{array}$ \\
\hline-15 & $249 \pm 29$ & $201 \pm 53$ & $297 \pm 108$ & $167 \pm 29$ & $298 \pm 68$ \\
0 & $209 \pm 20$ & $221 \pm 57$ & $257 \pm 91$ & $176 \pm 35$ & $275 \pm 68$ \\
30 & $141 \pm 18$ & $137 \pm 19$ & $176 \pm 44$ & $149 \pm 15$ & $237 \pm 36$ \\
60 & $170 \pm 29$ & $154 \pm 18$ & $207 \pm 16$ & $181 \pm 12$ & $198 \pm 40$ \\
90 & $133 \pm 25$ & $143 \pm 20$ & $167 \pm 17$ & $159 \pm 17$ & $200 \pm 16$ \\
120 & $160 \pm 21$ & $143 \pm 18^{\mathrm{a}}$ & $156 \pm 25$ & $190 \pm 31^{\mathrm{a}}$ & $207 \pm 17$ \\
150 & $169 \pm 32$ & $140 \pm 21^{\mathrm{c}}$ & $212 \pm 48^{\mathrm{b}}$ & $162 \pm 17^{\mathrm{b}}$ & $217 \pm 23$ \\
180 & $176 \pm 44$ & $137 \pm 12$ & $246 \pm 111$ & $174 \pm 24$ & $204 \pm 29$ \\
210 & $177 \pm 46$ & $103 \pm 22$ & $234 \pm 73$ & $264 \pm 65$ & $230 \pm 37$ \\
240 & $190 \pm 62$ & $130 \pm 12$ & $334 \pm 65$ & $374 \pm 126$ & $289 \pm 56$ \\
300 & $161 \pm 37$ & $170 \pm 38$ & $452 \pm 105$ & $558 \pm 178$ & $384 \pm 77$ \\
360 & $270 \pm 93$ & $203 \pm 48$ & $452 \pm 118$ & $556 \pm 197$ & $379 \pm 65$ \\
420 & $361 \pm 134$ & $307 \pm 127$ & $530 \pm 139$ & $514 \pm 102$ & $375 \pm 65$ \\
480 & $420 \pm 167$ & $397 \pm 85$ & $372 \pm 112$ & $472 \pm 117$ & $387 \pm 51$ \\
\hline a 6; b $5 ;$ c 3 indicate time of drop outs and number of patients who
\end{tabular}
finished the protocol

Table 3. Mean \pm SEM serum concentrations of non-esterfied free fatty acids $(\mu \mathrm{mol} / \mathrm{l})$

\begin{tabular}{rlllll}
\hline $\begin{array}{l}\text { Time } \\
\text { Min }\end{array}$ & $\mathrm{P}_{\mathrm{I}}$ & $\mathrm{P}_{\mathrm{II}}$ & $\mathrm{P}_{\mathrm{III}}$ & \multicolumn{1}{l}{$\mathrm{P}_{\mathrm{IV}}$} & \multicolumn{1}{l}{$\mathrm{P}_{\mathrm{V}}$} \\
\hline-15 & $665 \pm 61$ & $683 \pm 83$ & $653 \pm 63$ & $714 \pm 101$ & $743 \pm 111$ \\
0 & $646 \pm 64$ & $620 \pm 70$ & $662 \pm 74$ & $707 \pm 77$ & $714 \pm 118$ \\
30 & $603 \pm 51$ & $622 \pm 73$ & $636 \pm 80$ & $685 \pm 66$ & $704 \pm 106$ \\
60 & $587 \pm 63$ & $553 \pm 58$ & $625 \pm 82$ & $670 \pm 80$ & $669 \pm 75$ \\
90 & $608 \pm 83$ & $620 \pm 78$ & $582 \pm 71$ & $622 \pm 73$ & $773 \pm 153$ \\
120 & $625 \pm 98$ & $617 \pm 61^{\mathrm{a}}$ & $589 \pm 52$ & $656 \pm 78^{\mathrm{a}}$ & $734 \pm 109$ \\
150 & $692 \pm 94$ & $551 \pm 66^{\mathrm{c}}$ & $515 \pm 67^{\mathrm{b}}$ & $733 \pm 122^{\mathrm{b}}$ & $670 \pm 130$ \\
180 & $698 \pm 109$ & $525 \pm 47$ & $541 \pm 68$ & $775 \pm 142$ & $642 \pm 123$ \\
210 & $671 \pm 95$ & $549 \pm 87$ & $612 \pm 49$ & $836 \pm 184$ & $797 \pm 132$ \\
240 & $696 \pm 91$ & $572 \pm 67$ & $696 \pm 41$ & $969 \pm 231$ & $765 \pm 127$ \\
300 & $777 \pm 67$ & $705 \pm 70$ & $738 \pm 57$ & $1558 \pm 214$ & $833 \pm 164$ \\
360 & $781 \pm 85$ & $707 \pm 21$ & $676 \pm 39$ & $1059 \pm 212$ & $846 \pm 170$ \\
420 & $786 \pm 67$ & $784 \pm 124$ & $697 \pm 441$ & $1001 \pm 168$ & $860 \pm 175$ \\
480 & $927 \pm 77$ & $719 \pm 111$ & $710 \pm 77$ & $1165 \pm 195$ & $1012 \pm 190$ \\
\hline 6; b 5; 3 indicate time of drop outs and number of patients who \\
finished the protocol
\end{tabular}




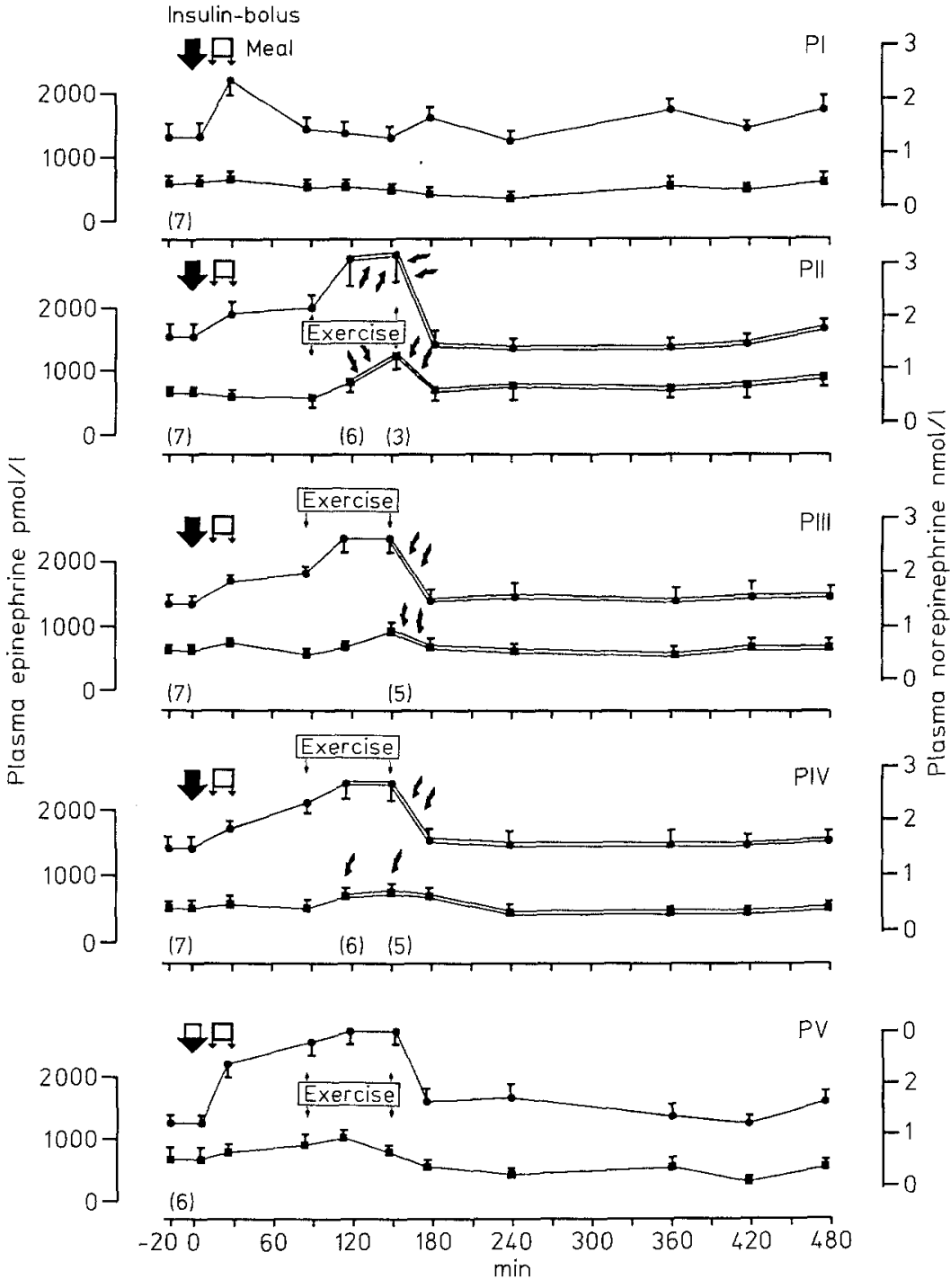

Fig.4. Plasma adrenaline and noradrenaline concentrations of CSII treated Type 1 diabetic patients before, during and after mild to moderate exercise in the five different protocols. Plasma epinephrine - plasma norepinephrine of ketone body concentration was observed in all protocols. No significant changes were obtained during exercise and in the early post-exercise phase, from $240 \mathrm{~min}$ onwards, a consistent increase was seen similarly in all protocols. This increase was probably due to the ongoing abstention from food by the subjects and was found to be most pronounced in protocol $P_{I V}$ where in addition, the serum free insulin concentrations were in a low range.

Mean serum concentrations of non-esterified fatty acids (NEFA; Table 3) ranged between 620 and $714 \mu \mathrm{mol} / \mathrm{l}$ during the base-line period of all the protocols. Following exercise NEFA increased consistently in all protocols, reaching significantly higher levels at $480 \mathrm{~min}$ as compared to base-line levels in $\mathrm{P}_{\mathrm{I}}$ and $\mathrm{P}_{\mathrm{V}}(p<0.01$, $p<0.05$ ). Base-line values of mean blood concentrations of pyruvate ranged between $60 \pm 10$ and $80 \pm 9 \mu \mathrm{mol} / 1$ in the different protocols. The pattern of pyruvate levels, seen throughout all the exercise protocols, was characterized by increases to maximal values at $60 \mathrm{~min}$ followed by a steady decrease to base-line levels at $240 \mathrm{~min}$ without further change. Under resting conditions blood lactate concentrations ranged from $0.9 \pm 0.1$ to $1.1 \pm 0.1 \mathrm{mmol} / 1$ and, increased significantly by comparable amounts during all exercise periods in accordance with the moderate work load applied.

\section{Hormonal responses}

No differences between the five protocols were seen in base-line plasma concentrations of either norepinephrine or epinephrine (Fig.4). In $\mathrm{P}_{\mathrm{I}}$, the mean norepinephrine concentrations increased from $1.64 \pm 0.25$ to $2.4 \pm 0.2 \mathrm{nmol} / 1(p<0.05)$ within the first $30 \mathrm{~min}$ of the protocol and then returned to base-line levels at $90 \mathrm{~min}$. The early increase of norepinephrine during the initial $30 \mathrm{~min}$ was similar in all protocols. During exercise norepinephrine rose further reaching maximal levels at $150 \mathrm{~min}$ and returned to base-line values thereafter. Plasma epinephrine concentrations starting at a mean of $442 \pm 65.5 \mathrm{pmol} / 1$ remained virtually unchanged throughout $\mathbf{P}_{\mathrm{I}}$ but rose during exercise reaching maximal values at 120 and $150 \mathrm{~min}$. These increases, however, were less pronounced than those of norepinephrine levels. 

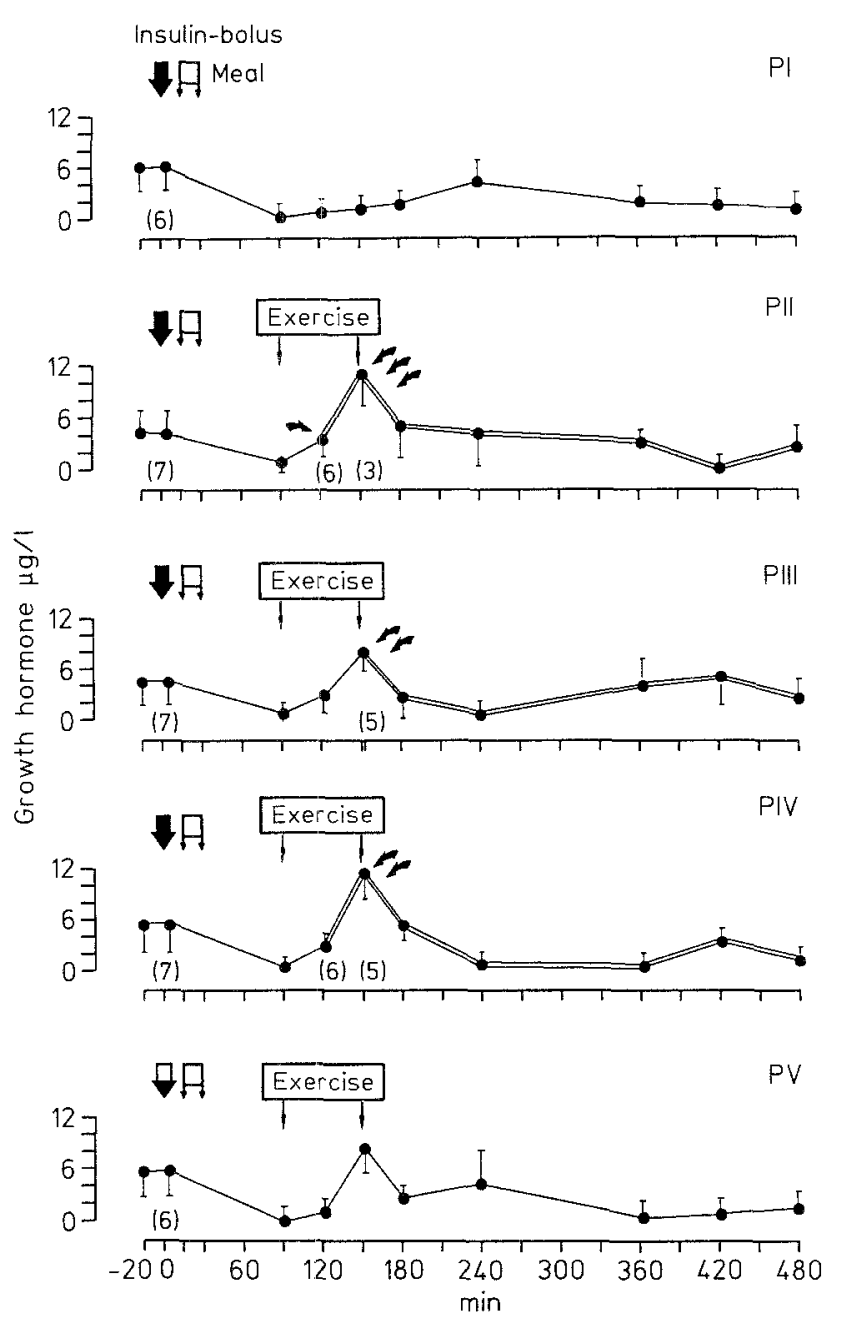

Fig.5. Serum growth hormone concentrations of CSII treated Type 1 diabetic patients before, during and after mild to moderate exercise in the five different protocols. Symbols as in Fig. 2

After an initial decrease from base-line (range: 4.3 to $6.3 \mu \mathrm{g} / \mathrm{l}$ ) to $90 \mathrm{~min}$ values (range: 0.4 to $0.6 \mu \mathrm{g} / \mathrm{l}$ ), mean serum growth hormone concentrations (Fig. 5) responded to exercise with an acute increase resulting in maximal values at the end of exercise (range: 8.2 to $11.9 \mu \mathrm{g} / \mathrm{l}$ ). This increase was similar in $\mathrm{P}_{\mathrm{II}}$ to $\mathrm{P}_{\mathrm{V}}$; thereafter, growth hormone concentrations declined to values in the range of 1.2 and $3.4 \mu \mathrm{g} / \mathrm{l}$. Plasma glucagon and serum cortisol concentrations did not differ among protocols under basal conditions and did not increase during or after the exercise periods.

\section{Discussion}

This study was performed to find an estimate of the insulin dosage adjustments necessary to prevent exercise induced hypoglycaemia in stable, (near)normoglycaemic Type 1 diabetic patients treated with CSII. Their stable metabolic situation was documented by concentrations of metabolites and counterregulatory hormones being comparable to those of normal subjects $[1,22]$. The nearly twofold rise of plasma norepinephrine and the less pronounced rise of epinephrine during exercise were comparable to those observed in CSII-treated diabetic patients [23] and in nondiabetic individuals performing graded exercise [24]. As in normal subjects [1], serum growth hormone levels showed about an eightfold increase during exercise. These catecholamine and growth hormone responses are likely to be induced by exercise rather than by low blood glucose during exercise since the changes observed in $P_{V}$ did not differ from those in the other protocols with lower levels of glycaemia. Mild to moderate exercise over 60 min was not associated with significant changes in plasma glucagon and serum cortisol concentrations indicating that one hour of moderate exercise does not stimulate secretion of these hormones.

The results of our study demonstrate that CSII-treated Type 1 diabetic patients at (near)normoglycaemia are likely to develop hypoglycaemia if they perform mild to moderate endurance exercise one hour after a meal without changing the insulin infusion rate and/or the premeal insulin bolus. This effect of exercise on blood glucose concentrations could be shown previously in CSII-treated patients [12-16]. However, as in normal subjects blood glucose levels appear to rise in Type 1 diabetic patients after a short bout of exercise as shown recently [25]. The hypoglycaemia in response to exercise varies partially depending on the intensity and duration of exercise, the alimentary status of the patients and the time interval between meal and exercise. As in the present study, a sharp fall in glycaemia was observed after 20 to $40 \mathrm{~min}$ of exercise started 90 min postprandially $[12,14]$. When the patients began to exercise 2 or $3 \mathrm{~h}$ postprandially no significant decrease of blood glucose levels could be found [16]. Exercising in the fasting state led to a strong blood glucose lowering effect in CSII treated patients in one study [15] whereas under similar conditions no significant changes in blood glucose concentrations were seen by others [13]. In none of these studies was the possibility of reducing the basal and/or premeal insulin infusion rates to prevent hypoglycaemia during exercise considered. The need to reduce the insulin dose in association with exercise has been shown already in conventionally treated patients $[4,8]$ and is further emphasized by our observation that catecholamines and growth hormone responses to exercise were not sufficient to prevent the exercise induced hypoglycaemia. Also, no information has hitherto been provided regarding the occurrence of late hypoglycaemic reactions, since the post exercise observation periods were short $(60-90 \mathrm{~min})$ and extended to $150 \mathrm{~min}$ in one study only [16].

The present results show that the risk of exercise-induced hypoglycaemia can be reduced by discontinuing the basal insulin infusion rate during exercise. However, this measure did not totally prevent acute hypoglycaemia. Any hypoglycaemic reactions during exercise in these patients could be avoided by reducing the premeal insulin bolus in addition to interrupting the basal insulin infusion rate. After administration of the reduced insulin bolus the rise in serum free insulin levels was attenuated resulting in substantially lower serum insulin levels at the beginning of exercise as compared to all other conditions in this study. This reduced insulin concentration at the start of the exer- 
cise was probably the main reason for the prevention of hypoglycaemia during exercise. It is even possible that solely the reduction of the premeal insulin bolus might have been sufficient to prevent hypoglycaemia during exercise in these patients. In daily life however, exercise may last longer than one hour and under such conditions it may be essential to stop the insulin infusion. In any case, patients planning to exercise within 1 to $2 \mathrm{~h}$ of a main meal for more then $30 \mathrm{~min}$ should be advised to take both precautions to prevent acute exercise induced hypoglycaemia, i.e. reduce the premeal bolus and interrupt the basal insulin infusion rate for the exercise period. If patients decide spontaneously to exercise within 1 to $2 \mathrm{~h}$ of a meal and administration of the insulin bolus, they still have the possibility of interrupting the insulin infusion to reduce the risk of hypoglycaemia. Under this condition hypoglycaemia during exercise may be prevented by consumption of additional amounts of carbohydrate prior to and/or during the physical activity.

Late-onset hypoglycaemic reactions after physical activity are not uncommon in tightly controlled diabetic patients [26, 27], as the enhancement of insulin sensitivity extends beyond the exercise period [28]. Exercise performed during late afternoon or evening hours carries a particular risk of nocturnal hypoglycaemia. Therefore, we have studied the effects of various basal insulin infusion rates for a period of $5 \mathrm{~h} 30 \mathrm{~min}$ after termination of the exercise. The infusion of the usually applied $100 \%$ basal rate after exercise was associated with blood glucose levels between 3.3 and $3.9 \mathrm{mmol} / \mathrm{l}$ and high free insulin levels in those three patients who were able to finish the study without hypoglycaemia. When interrupted during the exercise period, this insulin infusion rate was associated with normoglycaemia during the early post exercise period, while blood glucose tended to fall towards the end of the post-exercise period. The latter observation seems to be of particular importance if physical activity is performed during evening hours, which is likely to be the case with many patients. As a consequence low blood glucose levels and even hypoglycaemia must be expected during the night if the insulin infusion rate is not changed after exercise. A reduction of the basal insulin infusion rate by $25 \%$ after exercise was associated with stable near normoglycaemia while a more pronounced reduction of the infusion rate resulted in an undesirable deterioration of metabolic control.

In conclusion, Type 1 diabetic patients treated with continuous subcutaneous insulin infusion at (near)normoglycaemia need to reduce their insulin dosage before during and after mild to moderate endurance exercise in order to minimize the risk of acute and late hypoglycaemia. This recommendation should provide guidelines to guarantee the safety of CSII-treated subjects undertaking spontaneous physical activity during their everyday life.

Acknowledgements. We are indebted to Drs. A.A.R.Starke, R. Hehrmann and K. H. Kley for determination of plasma glucagon, serum growth hormone and serum cortisol respectively; and to Ms. C. Broermann and $\mathrm{H}$. Bartels for expert laboratory assistance. The study was supported by the Minister für Wissenschaft und For- schung des Landes Nordrhein-Westfalen (grant N IV B5-9211.13) and by the Deutsche Forschungsgemeinschaft (grant No $13 /$ SFB 113-83).

\section{References}

1. Berger M, Berchtold P, Cüppers HJ, Drost H, Kley HK, Müller WA, Wiegelmann W, Zimmermann-Telschow $\mathrm{H}$, Gries FA, Krüskemper HL, Zimmermann H (1977) Metabolic and hormonal effects of muscular exercise in juvenile type diabetics Diabetologia 13: 355-365

2. Vranic M, Berger M (1979) Exercise and diabetes mellitus. Diabetes 28: 147-167

3. Richter EA, Galbo H (1986) Diabetes, insulin and exercise. Sports Med 3: 275-288

4. Kemmer FW, Berchtold P, Berger M, Starke A, Cüppers HJ, Gries FA, Zimmermann H (1979) Exercise induced fall of blood glucose in insulin treated diabetics unrelated to alteration of insulin mobilization. Diabetes 28: 1131-1137

5. Koivisto V, Felig P (1978) Effects of leg exercise on insulin absorption in diabetic patients. N Engl J Med 298: 77-83

6. Kemmer FW, Berger M (1983) Exercise and Diabetes Mellitus: Physical activity as a part of daily life and its role in the treatment of diabetic patients. Intern J Sports Med 4:77-88

7. Kemmer FW, Sonnenberg GE, Cüppers HJ, Berger M (1986) Prevention of exercise induced hypoglycaemia in diabetes. In: Serrano-Rios M, Lefèbvre PJ (eds) Diabetes 1985. Elsevier Amsterdam, pp 963-967

8. Sane T, Helve E, Pelkonen R, Koivisto VA (1988) The adjustment of diet insulin dose during long-term endurance exercise in Type 1 (insulin-dependent) diabetic men. Diabetologia 31:35-40

9. Kemmer FW (1990) Exercise in diabetic patients. In: Torg JS, Welsh RP, Shepard RJ (eds) Current therapy in sports medicine, 2.Decker, Toronto, pp 109-114

10. Schiffrin A, Parikh S (1985) Accommodating planned exercise in Type I diabetic patients on intensive treatment. Diabetes Care 8: 337-342

11. Poussier P, Zinman B, Marliss EB, Albisser M, Perlman K, Caron D (1983) Open-loop intravenous insulin waveforms for postprandial exercise in Type I Diabetes. Diabetes Care 6: 129-134

12. Koivisto V, Tronier B (1983) Postprandial blood glucose response to exercise in Type I diabetes: comparison between pump and injection therapy. Diabetes Care 6: 436-440

13. Gooch BR, Abumrad NN, Robinson RP, Petrik M, Campbell D, Crofford OB (1983) Exercise in insulin-dependent diabetes mellitus: the effect of continuous insulin infusion using the subcutaneous, intravenous and intraperitoneal sites. Diabetes Care 6: $122-128$

14. Viberti GC, Home PD, Bilous RW, Alberti KGMM, Dalton N, Keen H, Pickup JC (1984) Metabolic effects of physical exercise in insulin-dependent diabetics controlled by continuous subcutaneous insulin infusion or conventional insulin therapy. Acta Endocrinol 105: 515-520

15. Schiffrin A, Parikh S, Marliss EB, Desrosiers MM (1984) Metabolic response to fasting exercise in adolescent insulin-dependent diabetic subjects treated with continuous subcutaneous insulin infusion and intensive conventional therapy. Diabetes Care $7: 255-260$

16. Trovati M, Carta Q, Cavalot F, Vitali S, Passarino G, Rocca G, Emanuelli G, Lenti G (1984) Continuous subcutaneous insulin infusion and postprandial exercise in tightly controlled Type I (insulin dependent) diabetic patients. Diabetes Care 7:327-330

17. Jones NL, Campbell EJM (1982) Clinical exercise testing, 2nd edn. Saunders, Philadelphia

18. Hochella NJ (1974) L-lactate. Determination with automatic analysers. In: Bergmeyer HU (ed) Methods of enzymatic analysis, 2nd edn. Verlag Chemie, Weinheim; Academic Press, New York London, pp 1479-1483 
19. Sole MJ, Hussain MN (1977) A simple specific radioenzymatic assay for the simultaneous measurement of picogram quantities of norepinephrine, epinephrine and dopamine in plasma and tissues. Biochem Med 18: 301-307

20. McGee J, Allen KG (1974) Preparation of methyl esters from the saponifiable fatty acids in small biological specimens for gasliquid chromatographic analysis. J Chromatogr 100: 35-42

21. Nakagawa S, Nakayama H, Sasaki T, Yoshimo K, Yu Y, Shinozaki K, Aoki S, Mashimo K (1973) A simple method for determination of serum free insulin levels in insulin-treated patients. Diabetes 22: 590-600

22. Kemmer FW, Bisping R, Steingrüber HJ, Baar H, Hardtmann F, Schlaghecke R, Berger M (1986) Psychological stress and metabolic control in patients with Type I diabetes mellitus. N Engl J Med 314: 1078-1084

23. Tamborlane WV, Sherwin RS, Koivisto V, Hendler R, Genel M, Felig P (1979) Normalization of the growth hormone and catecholamine response to exercise in juvenile-onset diabetic subjects treated with a portable insulin infusion pump. Diabetes 28 : 785-788

24. Galbo H, Holst JJ, Christensen NJ (1975) Glucagon and plasma catecholamine response to graded and prolonged exercise in man. J Appl Physiol 38: 70-76

25. Mitchell TH, Gebrehiwot A, Schiffrin A, Leiter LA, Marliss EB (1988) Hyperglycemia after intense exercise in IDDM subjects during continous subcutaneous insulin infusion. Diabetes Care 11: $311-317$

26. Campaigne BN, Wallberg-Henriksson H, Gunnarsson R (1987) Glucose and Insulin response in relation to insulin dose and caloric intake $12 \mathrm{~h}$ after acute physical exercise in men with IDDM. Diabetes Care 10: 716-721

27. MacDonald MJ (1987) Postexercise late-onset hypoglycemia in insulin-dependent diabetic patients. Diabetes Care 10:584-588

28. Bogardus C, Thuillez P, Ravussin E, Vasquez B, Narimiga M, Azhar S (1983) Effect of muscle glycogen depletion on in vivo insulin action in man. J Clin Invest 72: 1605-1610

Received: 6 December 1989

and in revised form: 29 June 1990

\section{Berger, M.D.}

Department of Nutrition and Metabolism

(WHO Collaborating Center for Diabetes)

Heinrich-Heine Universität

Moorenstrasse 5

W-4000 Düsseldorf

FRG 